\title{
Research on an improved MAP Super-Resolution in the Intelligent Transportation system
}

\author{
Haitang CHEN ${ }^{1, a}$, Guoyong HUANG ${ }^{2, b}$, Yan YANG ${ }^{1}$ \\ ${ }^{1}$ Faculty of Information Engineering and Automation, Kunming University of Science and \\ Technology, Kunming, 650500, China \\ ${ }^{2}$ Engineering Research Center for Mineral Pipeline Transportation of Yunnan Province, Kunming, \\ 650500, China

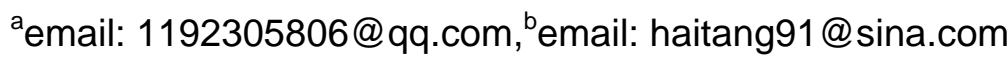

Keywords: MAP, Super-Resolution, Curvelet interpolation, Bicubic

\begin{abstract}
An improved MAP super-resolution was proposed aiming to the problem that the image dealt by traditional super-resolution was quite smooth and the marginal information was vanished. This algorithm constructs a $2 \times$ high resolution image from a low resolution image by Curvelet interpolation. The constructed image is divided into four directions and the corresponding directivity factor was calculated. Then, interpolate image by combining the directivity factor and its weight coefficient. And then, the interpolated image was interpolated by Bicubic interpolation. And the interpolated image was restored by MAP super-resolution. The experiment shows that this algorithm can remain the marginality of image better and restrain the appearance of blur and serration within the area where the margin and texture are rich compared to the traditional super-resolution algorithm.
\end{abstract}

\section{Introduction}

Surveillance video is one of the most important mediums in the realization of intelligent transportation [1]. The tracking or the recognition of vehicle is based on video with the development of intelligent transportation. A video with high quality have an important effect on the later processing which can reduce the difficulty of later processing and improve the efficiency. While, for the cost saving, the front-end video-capture devices are low resolution because of that the surveillance equipments should located in each area of city [2]. Thus, the detail section with important information will vanish. For instance, the model and license plate of vehicle causing the accident are difficult to recognize because of the low resolution. Therefore, the restoration of the image information from the existent image is very important and has attracted broad attention and research.

Traditional image restoration algorithm can eliminate the impact caused by disruptive factors during imaging procedure in some degree and improve the quality of image [3]. While the information of details vanished cannot be restored and the resolution of image cannot be improved essentially. Thus, the concept of super-resolution was proposed. The initial super-resolution construction which can improve the resolution of image without hardware device was proposed by Tsai and Huang, the cost of this algorithm is low and a great amount of image resource can be applied sufficiently. While, the algorithm proposed by Tsai and Huang is based on frequency domain which cannot deal the drawbacks of non-integral motion model. Then, plenty of algorithms based on space domain were proposed. For instance, the Projection onto Convex Set [4] (POCS) proposed by Stark et al. in 1989, the Iterative Back Projection [5] (IBP) proposed by Irani and Peleg in 1991, the ML-POCS [6] proposed in 1995 and the Maximum a Posterior [7] (MAP) proposed in 1997. Among them, MAP Super-Resolution Restoration Algorithm can regularize the distortion issue of super-resolution. This algorithm has been applied broadly in the image restoration because of its advantages such as the flexibility and variety of the chosen, the strong capacity of denoising and the uniqueness of solution [8]. Domestically, super-resolution restoration has attracted the attention of many experts and scholars. For instance, Binliang Jiao [9] et al. has 
proposed a super-resolution algorithm based on Wavelet interpolation. While, its precondition is that there should have a shift of one pixel which is hard to achieve without the knowledge of camera parameter. Wenbo $\mathrm{Li}$ [10] et al. have proposed a super-resolution algorithm based on Markov model and Shearlet transform, the writer applied it to a Synthetic Aperture Radar (SAR) image and have a good result. Rui Song [11] et al. has applied trilateral filtering regularization to the procedure of iteration within numerical solution under MAP frame. This algorithm can remain the margin, drop-off and the overall smooth features of image and have received a good result, but this algorithm didn't take the texture characteristics of image account. Jialin Tang [12] et al. has proposed a super-resolution algorithm combined with Wavelet theory, have achieved a good result and can remain the marginal characteristic better than traditional MAP Super-Resolution. But, this algorithm didn't take texture account which has result to the blur of textural margin.

To solve the problem of poor retainability of margin and texture in image cased by super-resolution restoration, this paper have proposed an improved MAP Super-Resolution Eeconstruction Algorithm which has combined the Curvelet interpolation with MAP Super-Resolution Restoration Algorithm. This algorithm constructs a $2 \times$ high-resolution image from a low resolution image by Curvelet interpolation and restored the image by MAP Super-Resolution Algorithm. The smoothness of margin caused by traditional MAP Super-Resolution can be solved by this algorithm. And it can remain the directional characteristic of image which can express the geometrical characteristic exist in the image commonly better.

The rest of this paper is organized as follows, section 2 describes the basic knowledge about MAP super-resolution and Curvelet interpolation, section 3 states the improved MAP super-resolution restoration algorithm, section 4 gives the experimental result of the algorithm proposed in this paper and section 5 is a conclusion.

\section{MAP super-resolution restoration image}

1. Curvelet Interpolation algorithm. Curvelet Interpolation Algorithm transforms the image from time domain to frequency domain by Curvelet transform, and frequency domain coefficient is divided into four directions. Then calculate the directivity factor, construct the weight coefficient of interpolation. Finally, interpolate the interpolation point which is divided into two kinds to get the high-resolution image [13].

Suppose $X$ is a $\mathrm{m} \times n$ low-resolution image, $Y$ is a $2 \mathrm{~m} \times 2 n$ high-resolution image and $Y$ is the image transformed from $X$ by $2 \times$ interpolation. As Figure 1 shows, the interpolation point is divided into two kinds according to its spatial position. The given points are located in the odd low and columns of high-resolution image $Y$ which are expressed by solid round. The first class of interpolation points is located in the even lows and columns of high-resolution image $Y$ which are expressed by solid triangle. The second class of interpolation points are located in the odd lows and even column and even lows and even columns of high-resolution image $Y$ which are expressed by hollow circle.

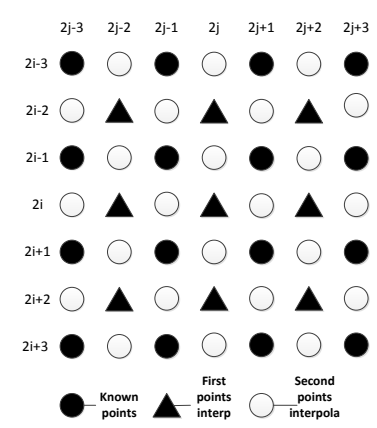

Fig.1. Classification of points interpolated in High-Resolution Image

Firstly, assign the value of pixels in image $X$ to the corresponding pixels in odd rows and column in image $Y$ as formula (1) shows [13]:

$Y(2 i-1,2 j-1)=X(i, j)$

Where $\mathrm{i}=1,2,3, \ldots m, j=1,2,3, \ldots n$. Then calculate four directivity factors $A_{1}, A_{2}, A_{3}$ and $A_{4}$, and interpolate 
the interpolating points by linear interpolation.

The first class of interpolating points are expressed by $Y(2 i, 2 j)$, calculate the estimated value in $45^{\circ}$ and $135^{\circ}$ of direction and calculate the estimated value of interpolating points by combining directivity factor with weight coefficient. The second class of interpolating points are expressed as $Y(x, y)$, where $\mathrm{y}=2 j$ when $\mathrm{x}=2 i-1, \mathrm{y}=2 j-1$ when $\mathrm{x}=2 i$. Then calculate the estimated value in horizontal and vertical direction and calculate the estimated value of interpolating points by combining directivity factor with weight coefficient.

By the two steps before, calculate the two classes of interpolation points $Y(2 i, 2 j)$ and $Y(x, y)$ in high-resolution image $Y$. The schematic diagram of the algorithm in this section is as Figure 2 shows.

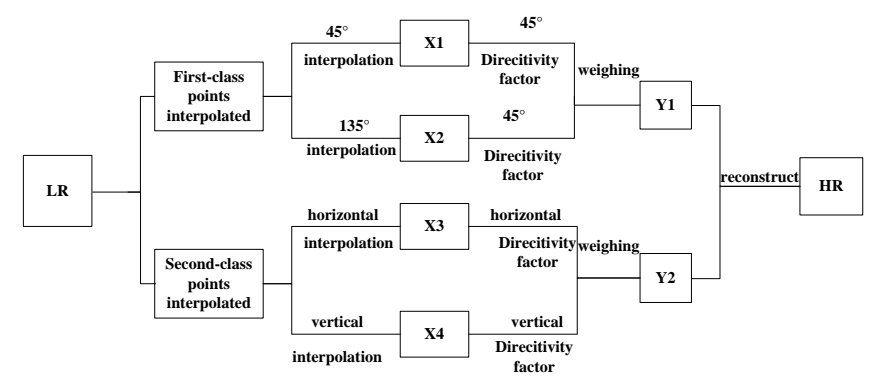

Fig.2. Schematic Diagram of $2 \times$ Interpolation of image based on Curvelet Transform

2. MAP Algorithm. MAP algorithm is a classical statistical method which turns the issue of super-resolution restoration to an issue of statistical estimation. MAP maximise the posterior probability of the arisen of high resolution with the knowledge of probability of the known low resolution image at the basic of Bayes principle.

The MAP estimation of unknown high resolution image $Z$ can be obtained as follows:

Miximise the conditional distribution density function $\operatorname{Pr}\left(z^{(k)} /\left\{y^{(l)}\right\}\right)$ of ideal image under the circumstance of the sequence of observatory image $\mathrm{y}=\left\{y^{(1)}, y^{(2)} \ldots y^{(k)}\right\}$. According to Bayes principle, the issue of optimal estimation of ideal high-resolution image can be expressed as formula (2) [3]:

$\wedge^{\wedge(k)}=\arg \max \operatorname{Pr}\left(z^{(k)} /\left\{y^{(1)}\right\}\right)=\arg \max \left(\frac{\left.\operatorname{Pr}\left(y^{(1)} /\left\{z^{(k)}\right\}\right) \operatorname{Pr}\left(z^{(k)}\right)\right)}{\operatorname{Pr}\left(y^{(1)}\right)}\right)$

The maximum posterior probability estimation can be expressed as formula bellow:

$$
\stackrel{\wedge}{z}^{(k)}=\arg _{z^{(k)}} \max \left\{\log \operatorname{Pr}\left(z^{(k)} / y^{\left(k-\frac{M-1}{2}\right)}, \ldots, y^{(k)}, \ldots, y^{\left(k+\frac{M-1}{2}\right)}\right)\right\}
$$

The maximization of $\operatorname{Pr}\left(z^{(k)} /\left\{y^{(l)}\right\}\right)$ is equivalent to the maximization of $\operatorname{Pr}\left(y^{(l)} /\left\{z^{(k)}\right\}\right) \operatorname{Pr}\left(z^{(k)}\right)$. The conditional distribution density function $\operatorname{Pr}\left(y^{(l)} /\left\{z^{(k)}\right\}\right)$ gives the error model generated at the process of the construction of sampling matrix under the condition of motion compensation. Assume that the error between frames is independent, and then the formula above is equivalent to the optimal problem as formula (4):

$$
\hat{\mathbf{Z}}^{(k)}=\arg _{z^{(k)}} \max \left\{\sum_{l=k-\frac{M-1}{2}}^{k+\frac{M-1}{2}} \log \operatorname{Pr}\left(y^{(l)} / z^{(k)}\right)+\log \operatorname{Pr}\left(z^{(k)}\right)\right\}
$$

The algorithm in this paper is the MAP Super-Resolution Restoration Algorithm which is achieved under the model of Huber-Markov. The maximum posterior probability estimation of high-resolution can be expressed as formula (5):

$$
\begin{aligned}
& \hat{\mathbf{z}}^{(k)}=\arg \min _{z^{k} \in \dot{f}} f\left(z^{(k)}\right)
\end{aligned}
$$

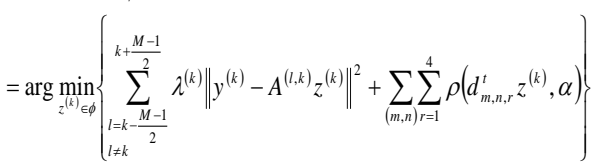

The definition of the constraint set $\phi$ is formula (6):

$$
\phi=\left\{\mathrm{z}^{(k)}: y^{(k)}=A^{(k, k)} \mathrm{z}^{(k)}\right\}
$$

Where $\mathrm{f}\left(\mathrm{z}^{(k)}\right)$ is objective function, $\alpha$ is the threshold parameter of Huber punishment function of edge. $\rho(\mathrm{x}, \alpha)$ will become quadratic punishment function of edge correspond to Gauss-Markov 
model when $\alpha$ trend to infinity. $\lambda^{(1, k)}$ is the corresponding trust parameter of $\mathrm{y}^{(l)}$ and $\mathrm{l} \neq k$. The relation of $\lambda^{(1, k)}$ is formula (7):

$$
\lambda^{(1, k)}=\frac{\beta}{\sigma^{(1, k) 2}}=\frac{\beta}{\sigma^{2}}
$$

The optimal solution of objective function $f\left(z^{(k)}\right)$ is unique because of the convex of Huber function. Finally, the estimation of high-resolution image can be obtained by using optimal algorithm.

\section{The Improved MAP Super-Resolution Restoration Algorithm}

The improved MAP Super-Resolution restoration algorithm proposed in this paper is applied to the super-resolution restoration of video image in transportation. The realization of the algorithm can be seem in Fig.3, the concrete steps are described as follws:

The noise generated during the acquirement of image may have a serious impact on the result of the algorithm. Thus, the image should be preprocessed before reconstruction to reduce the impact on the result of restoration.

Estimate the initialization of high-resolution image by Curvelet interpolation. Firstly, transform the image from time domain to frequency domain by Curvelet transform, and frequency domain coefficient is divided into four directions. Then, combining the weight coefficient with directivity factor and interpolate the interpolating points by linear interpolation. The estimation values are regarded as the reference of the reconstruction of image.

(1) Transform the pre-interpolation image from time domain to frequency domain by Curvelet transform, and frequency domain coefficient is divided into four directions. And calculate the directivity factors in $45^{\circ}, 135^{\circ}$, horizontal and vertical direction.

(2) Classify the interpolating points into two kinds and calculate their directivity factor. Then construct weight coefficient and combining it to interpolate all the points. Then the $\mathrm{m} \times n$ low-resolution image is turned to $2 \mathrm{~m} \times 2 n$ high-resolution image.

(3) The high-resolution image interpolated by Bicubic interpolation.

Restore the image by MAP algorithm.

(1) The prior information is expressed by probability density function based on Bayes principle and gets the issue of optimal estimation of ideal high-resolution image.

(2) The Huber-Markov model is introduced to this algorithm. And the estimation of high-resolution image is obtained by gradient descent.

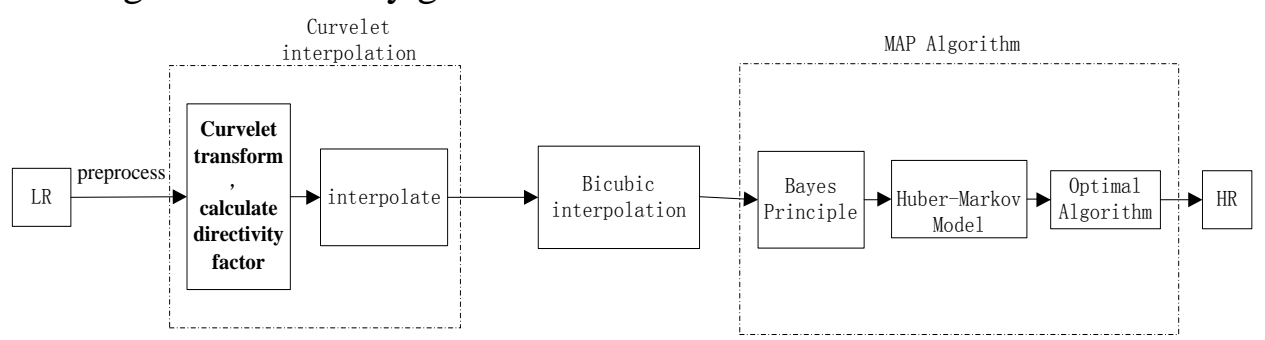

Fig.3. Schematic Diagram of improved MAP Algorithm

\section{Test results}

The experiment is operated on the image from the traffic video in this paper. The image is restored by linear interpolation and Curvelet interpolation under the frame of MAP Super-Resolution Restoration respectively. A series of video images whose quantity is 16 are sampled as Figure 4 shows. The first frame is regarded as a referential image and an initial image is obtained after motion estimation. Then construct a high-resolution image by linear interpolation and Curvelet interpolation. Then a high-resolution image is obtained by using MAP method. The image restoration is turned to an issue of optimal estimation by MAP algorithm and the high-resolution 
image is obtained by steepest descent.

Figure 5 and Figure 7 are the experimental result of linear interpolation, Curvelet interpolation and Curvelet interpolation combined with Bicubic interpolation under the frame of MAP Super-Resolution Restoration respectively. From the figure the conclusion that MAP Super-Resolution Restoration algorithm can restore the degrading image well and the majority of the lost information can be restored which can improve the resolution of degrading image in spatial domain is obvious. While, the feature of margin and texture of the image restored by MAP Super-Resolution restoration based on linear interpolation is not apparent and the result is not so perfect. And the image is divided into four directions in Curvelet interpolation algorithm and this algorithm take advantage of weight which can remain the feature of margin and texture of the image better. Thus, the algorithm combining Curvelet interpolation and MAP Super-Resolution Restoration proposed in this paper can keep the feature of margin and texture better which can express the ubiquitous geometric properties exist in image. While, the algorithm combined Curvelet interpolation and Bicubic interpolation based on MAP is brighter than other algorithms and it has less noise. The textured margin is more distinct and the restoration result is better which is closer to the ideal image, and it can reduce the difficult of the later processing.

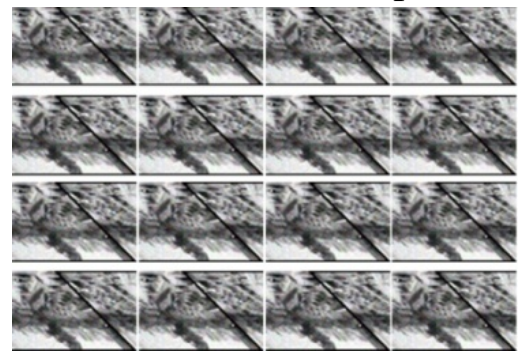

Fig.4. Multi-frame Image

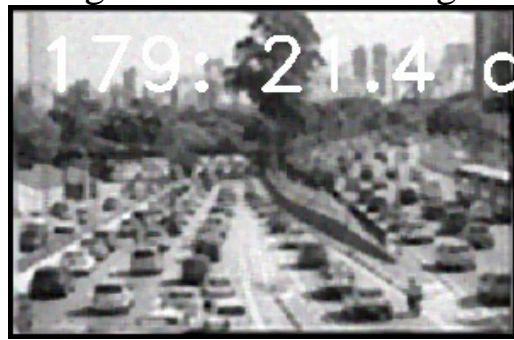

Fig.5. MAP Super-Resolution based on Linear Interpolation

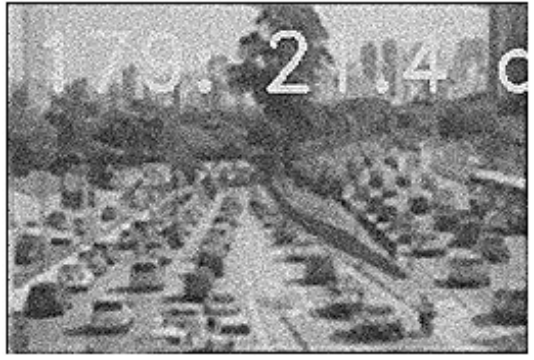

Fig.6. MAP Super-Resolution based on Curvelet Interpolation

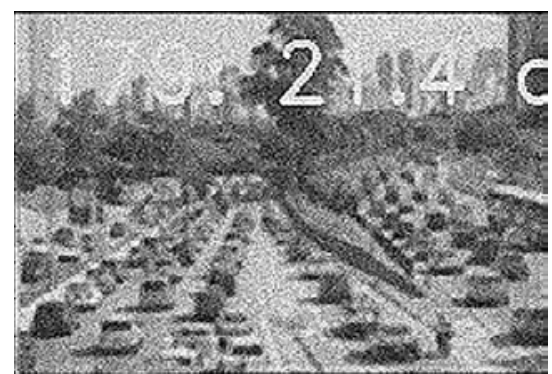

Fig.7. MAP Super-Resolution based on Curvelet Interpolation and Bicubic Interpolation 


\section{Conclusion}

The algorithm proposed in this paper combined Curvelet interpolation, Bicubic interpolation and MAP Super-Resolution Restoration has solved the issue of blur margin exist in traditional MAP Super-Resolution Restoration algorithm and has restrained the serration within the area where margin and texture are rich. The restoration result is good.

\section{Acknowledgement}

In this paper, the research was sponsored by is supported by the National Natural Science Founder of China (No. 51169007).

\section{References}

[1] H. Liu, Vehicle tracking and violation detection based on license plate location in high-definition image, Nanjing University of Science and Technology, Nanking, China, 2012.

[2] W. Xie, Research on the key technology of multi-frame Super-resolution image reconstruction, Wuhan University Press, Wuhan, China, 2014.

[3] L. Zhuo, S. Y. Wang, X. G. Li, Image/ Video Super Resolution, Posts \& Telecom Press, Peiking, China, 2011.

[4] Stark H, Oskui P, High-resolution image recovery from image-plane arrays using convex projections, Optical Society of America, 6(11) , 1715-1726, 1989.

[5] M Irani, S Peleg, Improving resolution by image registration, CVGIP: Graphical Models and Image Processing, 231-239, 1991.

[6] B. C. Tom, A. K. Katsaggelos, Reconstruction of a high-resolution image by simultaneous registration, restoration and interpolation of low-resolution images, Proc. of Int Conf. Image Processing, Washington D. C:IEEE, 539-542, 1995.

[7] R. C. Hardie, K. J. Barnard ,E. E. Armstrong, Joint MAP registration and high-resolution image estimation using a sequence of undersampled images, IEEE Transactions on Image Processing, 6(12), 1621-1633,1997.

[8] X. J. Yuan, K. Z. Wang, X. Z. Liu, Super-resolution based on MAP and edge preserving, Information Technology, (5), 45-47,2008.

[9] B. L. Jiao, W. L. Zhao, Application of wavelet and fractal theory on reconstruction of the image sequence with displacement, Optical Instruments, 27(6), 23-27, 2005.

[10] W. W. Li, W. Wu, D. S. Luo, H. B. Zhang , SAR Image Hallucination Based on Markov Model and Shearlet Transform, Journal of Sichuan University (Engineering Science Edition), 15(44), 101-108, 2012.

[11] R. Song, C. K. Wu, Y. Feng, Y. F. Zhang, A New MAP Based Texture Adaptive Super-Resolution Image Reconstruction Algorithm, Acta Electronic Sinica, 37(5), 1124-1129,2009.

[12] J. L. Tang, Z. F. Wu, C. G. Jiang, H. F. Sun, Study of Super-Resolution Image Restoration Algorithm Based on Wavelet Transform, Computer Science, 41(11), 147-149,2014.

[13] L. Zhao, H. J. He, Z. K. Yin, Texture direction adaptive image interpolation based on Curvelet Transform, Optoelectronics · Laser, 23(4), 798-803, 2012. 